\title{
On the structure of numerical sparse semigroups and applications to Weierstrass points
}

\author{
André Contiero ${ }^{\mathrm{a}, 1}$, Carlos Gustavo T. A. Moreira ${ }^{\mathrm{b}}$, Paula M. Veloso ${ }^{\mathrm{c}, 2}$ \\ ${ }^{a}$ Instituto de Matemática, Universidade Federal de Alagoas (UFAL). Avenida Lourival de Melo Mota, 57072-970, \\ Maceió - AL, Brazil \\ ${ }^{b}$ Instituto de Matemática Pura e Aplicada (IMPA). Estrada Dona Castorina 110, 22460-320, Rio de Janeiro - RJ, Brazil \\ ${ }^{c}$ Instituto de Matemática e Estatítica, Universidade Federal Fluminense (UFF). Rua Mário Santos Braga S/N, Campus \\ do Valonguinho, 24020-140 Niterói - RJ, Brazil
}

\begin{abstract}
In this work, we are concerned with the structure of sparse semigroups and some applications of them to Weierstrass points. We manage to describe, classify and find an upper bound for the genus of sparse semigroups. We also study the realization of some sparse semigroups as Weierstrass semigroups. The smoothness property of monomial curves associated to (hyper)ordinary semigroups presented by Pinkham and Rim-Vitulli, and the results on double covering of curves by Torres are crucial in this.
\end{abstract}

Keywords: numerical semigroups, genus, sparse semigroups, Weierstrass points, Weierstrass semigroups

2010 MSC: 20M13, 14H55

\section{Introduction}

Let $\mathcal{H}$ be a numerical semigroup of genus $g>1$. We say that $\mathcal{H}$ is a sparse semigroup if every two subsequent gaps of $\mathcal{H}$ are spaced by at most 2 . The concept of a sparse semigroup was introduced by Munuera-Torres-Villanueva in [MTV] and emerged as a generalisation of Arf semigroups. The latter appear naturally in the study of one-dimensional analytically unramified domains by analysing their valuation semigroups (see [Arf],n[BDF] and [Lip] for further details on Arf semigroups). Furthermore, one of the main subjects related to numerical semigroups are Weierstrass points on algebraic curves (points whose gap sequence of the numerical semigroup associated to a smooth projective pointed curve is the sequence of orders of vanishing of the holomorphic differentials of the curve at the base point.)

\footnotetext{
Email addresses: andrecontiero@mat.ufal.br (André Contiero), gugu@impa.br (Carlos Gustavo T. A. Moreira), pmveloso@id.uff.br (Paula M. Veloso)

${ }^{1}$ Partially supported by PPP-FAPEAL (20110901-011-0025-0048), by Programa de Pós-doutorado de Verão 2012 , IMPA, and by Projeto Universal CNPq (486468/2013-5)

${ }^{2}$ Partially supported by Programa Primeiros Projetos (CEX - APQ-03972-10)- FAPEMIG, by Programa de Pósdoutorado de Verão 2012, IMPA, and by Programa "Para Mulheres na Ciência" - L'Oréal, UNESCO, Academia Brasileira de Ciências
} 
In this work, we are concerned with studying the structure of sparse semigroups and some applications to Weierstrass points. Looking for a classification and for an upper bound for genus of sparse semigroups, we introduce a leap count assertion (Theorem 2.1), which involves an interplay between single and double leaps. Besides, it plays a fundamental role in the main results of this work.

Section 2 presents several consequences of Theorem 2.1. We prove that, if the genus of a sparse semigroup is large enough, then the last few gaps are spaced by 2 . This results is proved regardless the parity of the Frobenius number $\ell_{g}$ (Proposition 2.2). Additionally, we classify some sparse semigroups with few single leaps or with large Frobenius number. At this point, (hyper)ordinary semigroups (see [RV]) and $\gamma$-hyperelliptic semigroups show up (see [T]).

Looking for an upper bound for the genus of a sparse semigroup we introduce, in Section 3. the concept of a limit sparse semigroup: sparse semigroups with as many single as double leaps. Considering the parity of the Frobenius number, we classify limit sparse semigroups with even Frobenius number (Theorem 3.5), which are all hyperordinary with multiplicity 3. As a consequence, we get an upper bound for the genus of any sparse semigroup with even Frobenius number, namely $g<4 r$ where $\ell_{g}=2 g-2 r$ (Corollary 3.7).

We also classify limit sparse semigroups with odd Frobenius number. In this case, the multiplicity of the semigroup plays an import role. If the multiplicity of the limit sparse semigroup $\mathcal{H}$ is even, then $\mathcal{H}$ is an $r$-hyperelliptic semigroup, where $\ell_{g}=2 g-2 r-1$, (Theorem 3.8). On the other hand, if the multiplicity is odd, then either $\mathcal{H}$ is hyperordinary of multiplicity 3 , or a semigroup of multiplicity $2 r+1$, where $\ell_{g}=2 g-2 r-1$ is the Frobenius number of $\mathcal{H}$ (Theorem 3.9. With the classification of limit sparse semigroups with odd Frobenius number in mind, we find an upper bound for the genus of these sparse semigroups, namely $g \leq 4 r+1$, except when all nongaps smaller than the Frobenius number are even (Corollary 3.11).

Finally, in the last section, we study the realization of (limit) sparse semigroups as Weierstrass semigroups. At this point the smoothness property of monomial curves associated to (hyper)ordinary semigroups presented by Pinkham [P] and Rim-Vitulli [RV] is crucial. Furthermore, regarding $\gamma$-hyperelliptic sparse semigroups, the results by Torres [T] on double covering of curves are applied.

\section{Sparse semigroups}

Let $\mathbb{N}$ be the set of natural numbers. A numerical semigroup $\mathcal{H}=\left\{0=n_{0}<n_{1}<\ldots\right\} \subseteq \mathbb{N}$ of finite genus $g \geq 1$ is an additive subset of $\mathbb{N}$ containing 0 , closed under addition and such that there are only $g$ elements in the set $\mathbb{N} \backslash \mathcal{H}=\left\{1=\ell_{1}<\ell_{2}<\cdots<\ell_{g}\right\}$. The elements in $\mathbb{N} \backslash \mathcal{H}$ are called gaps and the largest gap $\ell_{g}$ is called the Frobenius number of $\mathcal{H}$. The elements of $\mathcal{H}$ are referred to as nongaps, and the smallest positive nongap is said to be the multiplicity of $\mathcal{H}$.

A sparse (numerical) semigroup $\mathcal{H}$ is a numerical semigroup where two subsequent gaps of $\mathcal{H}$ with $1 \leq \ell_{i-1}, \ell_{i} \in\left\{\ell_{1}<\ldots<\ell_{g}\right\}$ are spaced by at most 2 ,

$$
\ell_{i}-\ell_{i-1} \leq 2, i=2, \ldots, g, \ell_{i} \in \mathbb{N} \backslash \mathcal{H} \text {. }
$$

Equivalently, $\mathcal{H}$ is sparse if its first $c-g$ nongaps satisfy

$$
n_{i+1}-n_{i} \geq 2, i=1, \ldots, c-g,
$$

where $c:=\ell_{g}+1$ is the least integer such that $c+h \in \mathcal{H}$ for every $h \in \mathbb{N}$. The integer $c$ is said to be the conductor of $\mathcal{H}$ (clearly, $c=n_{c-g}$ ). 
Two particular classes of sparse semigroups will appear frequently in this work: ordinary sparse semigroups $\left(\mathcal{H}_{g}=\{0, g+1, g+2, \ldots\}\right)$ and hyperordinary sparse semigroups $(\mathcal{H}=$ $\left.m \mathbb{N}+\mathcal{H}_{g}, 0<m<g\right)$.

Another class of sparse semigroups are Arf semigroups [MTV, Corollary 2.2]. We recall that a numerical semigroup $\mathcal{H}$ is an Arf semigroup if $n_{i}+n_{j}-n_{k} \in \mathcal{H}$, for $i \geq j \geq k$ (see |BDF, Theorem I.3.4] for fifteen alternative characterizations of Arf semigroups, among which we call attention to the following: $\mathcal{H}$ is an Arf semigroup if and only if $2 n_{i}-n_{j} \in \mathcal{H}$, for all $i \geq j \geq 1$ ). There are, however, sparse semigroups that are not Arf see Remark 3.10 or [MTV, Example 2.3]).

It is well known that for any numerical semigroup the Frobenius number $\ell_{g}$ satisfies $\ell_{g} \leq$ $2 g-1$ (see, for instance, [0, Theorem 1.1]). We may, thus, define the parameter

$$
\kappa:=2 g-\ell_{g}>1,
$$

and we notice that $\kappa \leq g$.

Since sparse semigroups are the ones where subsequent gaps are either consecutive or spaced by 2 , it is only natural to count how many pairs of subsequent gaps are in either situation. Given a sparse semigroup $\mathcal{H}$, consider the sets:

$$
\begin{aligned}
\mathcal{D} & :=\left\{i ; \ell_{i+1}-\ell_{i}=2\right\} \text { ("double leaps"), } \\
\mathcal{S} & :=\left\{i ; \ell_{i+1}-\ell_{i}=1\right\} \text { ("single leaps"), }
\end{aligned}
$$

and their cardinalities:

$$
D:=\# \mathcal{D} \text { and } S:=\# \mathcal{S} \text {. }
$$

Theorem 2.1. Let $\mathcal{H}$ be a sparse semigroup of genus g. Then:

1. $D+S=g-1$.

2. $D=g-\kappa$.

3. $S=K-1$.

Proof. (1): Every gap, except the last one, $\ell_{g}$, is the starting point of a leap. So the total number of leaps, either single or double, is $g-1$. Thus, $D+S=g-1$.

(2) and (3): Between 1 and $\ell_{g}=2 g-\kappa$, there are $S$ single leaps and $D$ double leaps, regardless of their order. So, $\ell_{g}=2 g-\kappa=1+S+2 D$. This equation together with the previous one yield the desired results.

We shall denote leaps by an ordered pair of subsequent gaps $\left(\ell_{i}, \ell_{i+1}\right)$, where $\ell_{i}<\ell_{i+1}$. Clearly, a leap is single if $\ell_{i}+1=\ell_{i+1}$, and double if $\ell_{i}+2=\ell_{i+1}$.

Next proposition gives us a little bit more information on the structure of sparse semigroups. It tells us that, if $g \geq 2 \kappa-1$, then the last few gaps occur every two integers.

Proposition 2.2. Let $\mathcal{H}$ be a sparse semigroup of genus $g$. If $g \geq 2 \kappa-1$, then $\ell_{i+1}-\ell_{i}=2$, for every $i=2 \kappa-2, \ldots, g-1$.

Proof. If $g=2 \kappa-1$, then $\mathcal{H}$ has $\kappa-1$ single leaps. Let us assume that $\ell_{g}-\ell_{g-1}=1$. Thus $\ell_{g-1}-n_{i}, \ell_{g}-n_{i}$ are consecutive gaps for $i=1, \ldots, \kappa-1$. Since $\ell_{g-1}, \ell_{g}$ are consecutive gaps, the total number of single leaps for $\mathcal{H}$ is bigger than $\kappa-1$, which is a contradiction. Now, if $\mathcal{H}$ is a sparse semigroup of genus $g=2 \kappa+j$, with $j \in \mathbb{N}$. Then $\widetilde{\mathcal{H}}=\mathcal{H} \cup\left\{\ell_{g}\right\}$ is a sparse semigroup of genus $\widetilde{g}=2 \kappa+j-1$. Thus the gaps of $\mathcal{H}$ satisfy $\ell_{i+1}-\ell_{i}=2$, for $i=2 \kappa-2, \ldots, g-2$. Hence we just have to analyze $\ell_{g}-\ell_{g-1}$, which is analogous to the case where $g=2 \kappa-1$. 
For even values of $\kappa$,the previous result had been stated and proved by Munuera, Villanueva and Torres [MTV, Theorem 2.9 (3)] by means of a completely diverse approach.

Upon researching sparse semigroups, it became clear to us that those having genus $g=2 \kappa-1$ and Frobenius number $\ell_{g}=2 g-\kappa=3 \kappa-2$ are quite special. In fact, the lemma below suggests that they are "limit" in some sense; this notion will become clearer in the next section.

Lemma 2.3. Let $\mathcal{H}$ be a sparse semigroup of genus $g=2 \kappa+j, j \geq 0$, with Frobenius number $\ell_{g}=2 g-\kappa$. Then there is a sparse semigroup $\widetilde{\mathcal{H}}$ of genus $\widetilde{g}=2 \kappa-1$ and Frobenius number $\ell_{\widetilde{g}}=2 \widetilde{g}-\kappa=3 \kappa-2$ such $\mathcal{H}$ is a subsemigroup of $\widetilde{\mathcal{H}}$.

Proof. Since $g \geq 2 \kappa-1$, Proposition 2.2 tells us that the last $j+2$ gaps of $\mathcal{H}$ are spaced by 2. Consider the set $\widetilde{\mathcal{H}}=\mathcal{H} \cup\left\{\ell_{g-j-2}, \ell_{g-j-1}, \ldots, \ell_{g}\right\}$. Clearly, $\widetilde{\mathcal{H}}$ contains 0 and is additively closed. From the fact that $\mathcal{H}$ is sparse, we see that so is $\widetilde{\mathcal{H}}$. So $\widetilde{\mathcal{H}}$ is a sparse semigroup and, by construction, it has $\widetilde{g}=2 \kappa-1$ gaps, $\mathcal{H}$ is a subsemigroup of $\widetilde{\mathcal{H}}$, and its Frobenius number is $\ell_{\widetilde{g}}=2 \widetilde{g}-\kappa=3 \kappa-2$.

We present now several consequences and applications of our leap-count result (Theorem 2.1), which illustrate the techniques used in the theory of sparse semigroups. It will be clear, trough the next results, that there are only few sparse semigroups with large Frobenius number (or, equivalently, with few single leaps). We will make this statement more precise in the next section.

We recall that a numerical semigroup is said to be symmetric (resp. quasi-symmetric) if $\ell_{g}=2 g-1$ (resp. $\ell_{g}=2 g-2$ ).

Corollary 2.4. If $\mathcal{H}$ is a symmetric sparse semigroup, then $\mathcal{H}$ is the hyperelliptic semigroup $\mathcal{H}=\langle 2,2 g+1\rangle$.

Proof. Since $\ell_{g}=2 g-1$, the sparse semigroup $\mathcal{H}$ does not have single leaps i.e. $\kappa=0$. Then $2 \in \mathcal{H}$ and all the odd numbers between 1 and $\ell_{g}$ are gaps.

Corollary 2.5. If $\mathcal{H}$ is a quasi-symmetric sparse semigroup, then, either $\mathcal{H}=\langle 3,4,5\rangle$, or $\mathcal{H}=$ $\langle 3,5,7\rangle$.

Proof. Since $\ell_{g}=2 g-2$, we have that $\kappa=2$ and so $S=1$ and $D=g-2$. We must have $1,2 \notin \mathcal{H}$, which already accounts for the only single leap, so $3 \in \mathcal{H}$. Since all subsequent leaps must be double, the remaining gaps must all be even numbers. Then $g \leq 3$. Now, notice that there are no numerical semigroups of genus $g=1$, otherwise $\ell_{g}=\ell_{1}=2 g-2=0$, a contradiction. If $g=2$, we have $\ell_{g}=\ell_{2}=2 g-2=2$, and so $\mathcal{H}=\langle 3,4,5\rangle$. Finally, for $g=3$, we have $\ell_{g}=2 g-2=4$, so 1 and 2 are also gaps, for they divide 4 , and thus $\mathcal{H}=\langle 3,5,7\rangle$.

We say that a numerical semigroup is $\gamma$-hyperelliptic if it has exactly $\gamma$ even gaps. For the sake of clarity, we note that a $\gamma$-hyperelliptic semigroup may have odd gaps and the integer $\gamma$ is not necessarily its genus. Such semigroups are closely related with double covering of curves $[\mathrm{BC}, \mathrm{T}, \mathrm{T2}]$. Additionaly, they arise when we deal with the characterization of sparse semigroups having as many single as double leaps (see next section).

Theorem 2.6. Let $\mathcal{H}$ be a sparse semigroup having genus $g \geq 3$ and $\ell_{g}=2 g-3$. Then $\mathcal{H}$ is one of the following: 
1. $\mathcal{H}=3 \mathbb{N}+\mathcal{H}_{5}, \mathcal{H}$ is 2-hyperelliptic;

2. $\mathcal{H}=3 \mathbb{N}+\mathcal{H}_{7}, \mathcal{H}$ is 2-hyperelliptic;

3. $\mathcal{H}=2(\mathbb{N} \backslash\{1\}) \cup \mathcal{H}_{2 g-2}, \mathcal{H}$ is 1-hyperelliptic.

Proof. Theorem 2.1 (3) tells us that $S=2$. Clearly, $\ell_{1}=1$ and $\ell_{2}=2$, which accounts for one single leap. If $3 \in \mathcal{H}$, by the sparse property, we must have that $1,2,4,5 \notin \mathcal{H}$, and this accounts for all 2 single leaps. Thus, either $\mathbb{N} \backslash \mathcal{H}=\{1,2,4,5\}$ and $g=4(1)$, or $\mathbb{N} \backslash \mathcal{H}=\{1,2,4,5,7\}$ and $g=5$ (2). Otherwise, $\ell_{3}=3$, and all leaps from this point on must be double. So $\mathbb{N} \backslash \mathcal{H}=$ $\{1,2,3,5,7, \ldots 2 g-3\}$ and $\mathcal{H}=\{4,6, \ldots, 2 g-4\} \cup\{n \in \mathbb{N} ; n \geq 2 g-2\}$ (3).

Corollary 2.7. Let $\mathcal{H}$ be a numerical semigroup having genus $g \geq 6$ and $\ell_{g}=2 g-3$. Then the following are equivalent:

a. $\mathcal{H}$ is sparse;

b. $\mathcal{H}$ is 1-hyperelliptic.

Proof. The implication $a . \Longrightarrow b$. follows immediately from the previous theorem. The other implication can be proved as follows: since $\mathcal{H}$ is 1-hyperelliptic, the only even gap of $\mathcal{H}$ must be 2 . Indeed, if $\ell>2$ were an even gap, then, from $\ell=2+(\ell-2), 2$ or $\ell-2$ would be a smaller even gap, a contradiction. So, every even number larger than 2 is a nongap, and thus $\ell_{r} \geq 2 r-3, \forall r \geq 3$, and, if the equality holds for $r=g$, it must hold for every $r$ with $3 \leq r \leq g$, which implies that $\mathcal{H}$ is sparse.

Theorem 2.8. Let $\mathcal{H}$ be a sparse semigroup having genus $g \geq 4$ and $\ell_{g}=2 g-4$. Then $\mathcal{H}$ is one of the following:

1. $\mathcal{H}=3 \mathbb{N}+\mathcal{H}_{8}$, and $\mathcal{H}$ is 3-hyperelliptic;

2. $\mathcal{H}=3 \mathbb{N}+\mathcal{H}_{10}$, and $\mathcal{H}$ is 4-hyperelliptic;

3. $\mathcal{H}=4 \mathbb{N}+\mathcal{H}_{6}$, and $\mathcal{H}$ is 2-hyperelliptic;

4. $\mathcal{H}=\mathcal{H}_{4}$, and $\mathcal{H}$ is 2-hyperelliptic;

5. $\mathcal{H}=5 \mathbb{N}+\mathcal{H}_{6}$, and $\mathcal{H}$ is 3-hyperelliptic;

6. $\mathcal{H}=\{0,5,7\} \cup \mathcal{H}_{8}$, and $\mathcal{H}$ is 4-hyperelliptic;

Proof. Theorem 2.1 (3) tells us that $S=3$. Again, $\ell_{1}=1$ and $\ell_{2}=2$, which account for one single leap.

If $3 \in \mathcal{H}$, since $\mathcal{H}$ is sparse and $S=3$, we must have that $1,2,4,5,7,8 \notin \mathcal{H}$ accounting for all 3 single leaps. Thus, either $\mathbb{N} \backslash \mathcal{H}=\{1,2,4,5,7,8\}$ and $g=6(1)$, or $\mathbb{N} \backslash \mathcal{H}=\{1,2,4,5,7,8,10\}$ and $g=7(2)$.

Otherwise, $\ell_{3}=3$, which accounts for a second single leap. If $4 \in \mathcal{H}$, since $\mathcal{H}$ is sparse, $5 \notin \mathcal{H}$. Notice that $6 \notin \mathcal{H}$ (otherwise, every even number $n \geq 4$ is in $\mathcal{H}$, and we would only have 2 single leaps, a contradiction). So we have the remaining single leap $(5,6)$, and all leaps from his point on must be double. On the other hand, we should have $8=4+4 \in \mathcal{H}$. Thus, $\mathbb{N} \backslash \mathcal{H}=\{1,2,3,5,6\}(3)$.

Finally, if $4 \notin \mathcal{H}$, then all 3 single leaps occur on gaps $1,2,3,4$ and all other leaps are double. Thus, $5 \in \mathcal{H}$ and the only possibilities are $\mathbb{N} \backslash \mathcal{H}=\{1,2,3,4\}(4), \mathbb{N} \backslash \mathcal{H}=\{1,2,3,4,6\}$ (5) and $\mathbb{N} \backslash \mathcal{H}=\{1,2,3,4,6,8\}(6)$. 
Notice that the previous result implies, in particular, that, if $\mathcal{H}$ is a sparse semigroup having genus $g \geq 4$ and $\ell_{g}=2 g-4$, then $g \leq 7$. We will see in the next section (Theorem 3.7) that it is possible to generalize this fact (and the result about quasi-symmetric sparse semigroups) in the sense that for any fixed $r$, there are only a finite number of (sorts of) sparse semigroups, which can be explicitly listed.

Theorem 2.9. Let $\mathcal{H}$ be a sparse semigroup having genus $g \geq 5$ and $\ell_{g}=2 g-5$. Then $\mathcal{H}$ is one of the following:

1. $\mathcal{H}=3 \mathbb{N}+\mathcal{H}_{11}$, and $\mathcal{H}$ is 4-hyperelliptic;

2. $\mathcal{H}=3 \mathbb{N}+\mathcal{H}_{13}$, and $\mathcal{H}$ is 4-hyperelliptic;

3. $\mathcal{H}=2(\mathbb{N} \backslash\{1,3\}) \cup \mathcal{H}_{2 g-4}$, with $g \geq 6$, and $\mathcal{H}$ is 2-hyperelliptic;

4. $\mathcal{H}=\{0,5,7\} \cup \mathcal{H}_{9}$, and $\mathcal{H}$ is 4-hyperelliptic;

5. $\mathcal{H}=\{0,5,7,10\} \cup \mathcal{H}_{11}$, and $\mathcal{H}$ is 4-hyperelliptic;

6. $\mathcal{H}=\{0,5,7,10,12\} \cup \mathcal{H}_{13}$, and $\mathcal{H}$ is 4-hyperelliptic;

7. $\mathcal{H}=\{0,5\} \cup \mathcal{H}_{7}$, and $\mathcal{H}$ is 3-hyperelliptic;

8. $\mathcal{H}=\{0,5,8\} \cup \mathcal{H}_{9}$, and $\mathcal{H}$ is 3-hyperelliptic;

9. $\mathcal{H}=\{0,5,8,10\} \cup \mathcal{H}_{11}$, and $\mathcal{H}$ is 4-hyperelliptic;

10. $\mathcal{H}=2(\mathbb{N} \backslash\{1,2\}) \cup \mathcal{H}_{2 g-4}$, with $g \geq 5$, and $\mathcal{H}$ is 2-hyperelliptic;

Proof. The proof technique is very similar to the one in the previous theorem.

Theorem 2.1 (3) tells us that there are $S=4$. Again, $\ell_{1}=1$ and $\ell_{2}=2$, which account for one single leap. If $3 \in \mathcal{H}$, since $\mathcal{H}$ is sparse, we must have that $1,2,4,5,7,8,10,11 \notin \mathcal{H}$, accounting for all 4 single leaps. Thus, either $\mathbb{N} \backslash \mathcal{H}=\{1,2,4,5,7,8,10,11\}$ and $g=8$ (1), or $\mathbb{N} \backslash \mathcal{H}=\{1,2,4,5,7,8,10,11,13\}$ and $g=9(2)$.

Otherwise, $\ell_{3}=3$, which accounts for a second single leap. If $4 \in \mathcal{H}$, since $\mathcal{H}$ is sparse, we must have that, for every $n \in 4 \mathbb{N}, n<\ell_{g}$, both $n-1$ and $n+1$ are gaps. In particular, $5,7 \notin \mathcal{H}$. Notice that $6 \notin \mathcal{H}$ (otherwise, every even number $n \geq 4$ is in $\mathcal{H}$, and we would only have 2

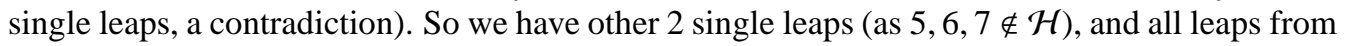
this point on must be double. Thus, $\mathbb{N} \backslash \mathcal{H}=\{1,2,3,5,6,7,9,11, \ldots, 2 g-5\}$ (and $2 g-5 \geq 7$, so $g \geq 6$ ) (3).

Otherwise, $\ell_{4}=4$, which accounts for a third single leap. If $5 \in \mathcal{H}$, since $\mathcal{H}$ is sparse and $5<\ell_{g}, 6 \notin \mathcal{H}$ and exactly one of the following possibilities holds: $7 \notin \mathcal{H}$ or $8 \notin \mathcal{H}$ (the two cannot happen simultaneously, as we would then have 6 single leaps).

If $7 \in \mathcal{H}$, then $8 \notin \mathcal{H}$ and, since $10=5+5 \in \mathcal{H}$ and $9 \leq \ell_{g}, 9 \notin \mathcal{H}$. This accounts for the fourth and last single leap of $\mathcal{H}$. So all leaps from his point on must be double, which means that all even numbers greater than 9 must be in $\mathcal{H}$. Since $14,15 \in \mathcal{H}$ and $\mathcal{H}$ is sparse, $\{n \geq 14\} \subset \mathcal{H}$. Thus, in this case, we have the following possibilities for $\mathcal{H}: \mathcal{H}=\{0,5,7\} \cup\{n \geq 10\}$ (4), $\mathcal{H}=\{0,5,7,10\} \cup\{n \geq 12\}(2)$ and $\mathcal{H}=\{0,5,7,10,12\} \cup\{n \geq 14\}$ (6).

If $8 \in \mathcal{H}$, then $7 \notin \mathcal{H}$, and all single leaps occur on gaps $1,2,3,4,6,7$. Thus, all leaps from this point on must be double, which means that all even numbers greater than 7 must be in $\mathcal{H}$. So $13=5+8 \in \mathcal{H}$, and thus, in this case, we have the following possibilities for $\mathcal{H}$ : $\mathcal{H}=\{0,5\} \cup\{n \geq 8\}(7), \mathcal{H}=\{0,5,8\} \cup\{n \geq 10\}(8)$ and $\mathcal{H}=\{0,5,8,10\} \cup\{n \geq 12\}$ (9).

On the other hand, if $5 \notin \mathcal{H}$, then all 4 single leaps occur on gaps 1,2, 3, 4, 5 and all other leaps are double. Thus,

$$
\mathbb{N} \backslash \mathcal{H}=\{1,2,3,4,5,7,9, \ldots, 2 g-5\}
$$

(here $2 g-5 \geq 5$, and so $g \geq 5$ ) (10). 
An important feature of Theorems 2.6 and 2.9 will be generalized in the next section (Corollary 3.11): if $\mathcal{H}$ is a sparse semigroup having genus $g$ for which $\ell_{g}=2 g-(2 r+1)$, where $r \in \mathbb{N}$, then, if $g>4 r+1$, all nongaps of $\mathcal{H}$ smaller than $\ell_{g}$ are necessarily even. In this case all the $\left(\ell_{g}+1\right) / 2=g-r$ odd positive integers smaller than $\ell_{g}+1$ are gaps, so there are $r$ even gaps (i.e., $\mathcal{H}$ is $r$-hyperelliptic), and the set $\{m \in \mathbb{N} ; 2 m \in \mathcal{H}\}$ is a semigroup of genus $r$.

Theoretically, one could use the proof technique in Theorems 2.6 and2.9 (and/or the above remark) to characterize all sparse semigroups having $\ell_{g}=2 g-(2 r+1)$ for any fixed $r \in \mathbb{N}$. However, as $r$ grows, the number of cases to be analyzed quickly add up, and the arguments in the proof become more intricate.

\section{Limit sparse semigroups}

Having in mind the results of the previous section, it is only natural for one to ask about the existence of sparse semigroups of genus $g \geq 4 r-1$ and Frobenius number $\ell_{g}=2 g-2 r$ (where $\kappa=2 r$ ). A preliminary analysis of examples suggests that such semigroups do not exist if $g>4 r-1$. This fact together with Lemma2.3 reinforces the idea that sparse semigroups of genus $g=4 r-1$ with even Frobenius number $\ell_{g}=2 g-2 r$ are special.

Remark 3.1. We note that Theorem 2.1 assures us that a sparse semigroup has genus $g=2 \kappa-1$ if and only if $S=D$.

We call a sparse semigroup with as many single as double leaps (equivalently, of genus $g=2 \kappa-1)$ a limit sparse semigroup.

Starting from searching for an upper bound to the genus of limit sparse semigroups with even Frobenius number, our aim is to analyze more closely the structure of such semigroups regardless of its Frobenius number's parity. When needed, we shall denote $\ell_{g}=2 g-2 r(\kappa=2 r)$ for even Frobenius numbers, or $\ell_{g}=2 g-2 r-1(\kappa=2 r+1)$ for odd Frobenius numbers.

Lemma 3.2. If $\mathcal{H}$ is a limit sparse semigroup, then $\#\left\{\mathcal{H} \cap\left\{1,2,3, \ldots, \ell_{g}\right\}\right\}=S=D$.

Proof. Clearly, there are $\ell_{g}=2 g-\kappa$ natural numbers in the set $\left\{1, \ldots, \ell_{g}\right\}, g$ of which are gaps. So, the $g-\kappa$ remaining ones are all in $\mathcal{H} \cap\left\{1, \ldots, \ell_{g}\right\}$ and, thus, $\#\left\{\mathcal{H} \cap\left\{1,2,3, \ldots, \ell_{g}\right\}\right\}=g-\kappa=$ $D=S$.

Lemma 3.3. If $\mathcal{H}$ is a limit sparse semigroup with even Frobenius number $\ell_{g}=2 g-2 r$. Then $4 \notin \mathcal{H}$.

Proof. We know that $\ell_{g}=6 r-2, \ell_{g-1}=6 r-4$, so $3 r-2,3 r-1$ are also gaps. Assume, by contradiction, that $4 \in \mathcal{H}$, then, analyzing the residual class of $r$ modulo 4 , one of the integers $6 r-2,3 r-2,3 r-2$ is a nongap, a contradiction.

Let us now see that for each $r$ there is precisely one limit sparse semigroup with even Frobenius number. First, we state and prove another technical lemma:

Lemma 3.4. Let $\mathcal{H}$ be a limit sparse semigroup with even Frobenius number $\ell_{g}=2 g-2 r=$ $6 r-2$. Then $3 \in \mathcal{H}$ if and only if $6 r-5 \notin \mathcal{H}$. 
Proof. It is clear that if $3 \in \mathcal{H}$ then $\mathcal{H}=3 \mathbb{N}+\mathcal{H}_{6 r-2}$, so $6 r-5 \notin \mathcal{H}$.

Now, let us assume that $6 r-5 \notin \mathcal{H}$. Since $S=D$ and because of Lemma 3.2, there are $2 r-2$ nongaps in the interval $[1,6 r-5]$. Then for each such nongap $n \in \mathcal{H}$, the consecutive numbers $6 r-5-n$ and $6 r-4-n$ are gaps, which produces $2 r-2$ single leaps. Notice that the single leaps $(6 r-5-n, 6 r-4-n)$ are disjoint, for every $n \in \mathcal{H}$, because of the sparse property.

Suppose, by contradiction, that $3 \notin \mathcal{H}$. Thus $(1,2)$ and $(2,3)$ are single leaps, and $(6 r-5-$ $n, 6 r-4-n)$ are $2 r-2$ disjoint single leaps, for every $n \in \mathcal{H} \cap[1,6 r-5]$, and so at least one of the two single leaps $(1,2)$ and $(2,3)$ are not of this form. Hence, since $(6 r-5,6 r-4)$ is also a single leap, the number of single leaps is bigger than $2 r-1$, which is a contradiction.

Theorem 3.5. Let $\mathcal{H}$ be a limit sparse semigroup with even Frobenius number $\ell_{g}=2 g-2 r$. Then $\mathcal{H}=3 \mathbb{N}+\mathcal{H}_{6 r-2}$.

Proof. In view of Lemma 3.4 all we have to do is to show that $6 r-5$ is a gap. Suppose otherwise. So, there is an integer $x \geq 3$ such that the last single leap is $(6 r-2 x-1,6 r-2 x)$. So, in the interval [ $6 r-2 x, 6 r-2]$, all even numbers are gaps and all odd numbers are nongaps.

Let $n_{1}$ be the multiplicity of $\mathcal{H}$. We first notice that it cannot be that $n_{1}$ is an odd number and $n_{1}<2 x-1$. Otherwise, $6 r-2-n_{1}$ would be an odd gap and $6 r-2-n_{1}>6 r-2 x-1$, a contradiction. Moreover, it cannot be that $n_{1}$ is an even number and $n_{1} \leq 2 x$. Otherwise, there would be an even nongap, a multiple of $n_{1}$, between $6 r-2 x$ and $6 r-2$, which is also a contradiction. Then there are only two possibilities for $n_{1}$ :

(A) $n_{1}>2 x$

(B) $n_{1}=2 x-1$.

Since the nongaps in the interval $[6 r-2 x, 6 r-2]$ are the odd numbers, there are $2 r-x$ positive nongaps smaller than $6 r-2 x-1$. For each one of them, say $n \in \mathcal{H} \cap[1,6 r-2 x-2]$, $6 r-2 x-1-n$ and $6 r-2 x-n$ are consecutive gaps, producing $2 r-x$ single leaps.

Let us consider each case separately:

Consider (A). In this case, $1,2,3, \ldots, n_{1}-1$ are consecutive gaps. The single leaps $(6 r-$ $2 x-1-n, 6 r-2 x-n)$ are all disjoint for every $n \in \mathcal{H}, n<6 r-2 x-1$. Then, among the single leaps in the interval $\left[1, n_{1}-1\right]$, there are at least $x-1$ different from the ones in $\{(6 r-2 x-1-n, 6 r-2 x-n) ; n \in \mathcal{H}, n<6 r-2 x-1\}$. Since $(6 r-2 x-1,6 r-2 x)$ is also a single leap, we have, so far, $(x-1)+(2 r-x)+1=2 r$ single leaps, which is a contradiction, because $S=2 r-1$.

Now, let us suppose (B). Since $S=2 r-1$, there are exactly $x-2$ single leaps in the interval $\left[1, n_{1}-1\right]$ that are not in $\{(6 r-2 x-1-n, 6 r-2 x-n) ; n \in \mathcal{H}, n<6 r-2 x-1\}$. Thus, there are $x-1$ single leaps in the interval [1,2x-2] of the type $(6 r-2 x-1-n, 6 r-2 x-n)$, where $n \in \mathcal{H} \cap[1,6 r-2 x-2]$, which are necessarily $(1,2),(3,4), \ldots,(2 x-3,2 x-2)$. Hence $6 r-4 x+2, \ldots, 6 r-2 x-4,6 r-2 x-2 \in \mathcal{H}$. In particular, since $x \geq 3,6 r-2 x-4 \in \mathcal{H}$.

Now, consider the interval $[2 x-1,6 r-4 x+1]$ which has $3(2 r-2 x+1) \neq 0$ integers and contains $2 r-x-(x-1)=2 r-2 x+1$ nongaps. For each nongap $n \in \mathcal{H} \cap[2 x-1,6 r-4 x+1]$, we consider the consecutive gaps $6 r-2 x-1-n$ and $6 r-2 x-n$, which both belong to [2x-1, $6 r-4 x+1]$ (and thus are $2 r-2 x-1$ single leaps contained in this interval). Then, since $\mathcal{H}$ is sparse, the nongaps in the interval $[2 x-1,6 r-4 x+1]$ are necessarily the integers congruent to $2 x-1(\bmod 3)$. Hence, $2 x+2 \in \mathcal{H}$ (this is clear when the interval [ $2 x-1,6 r-4 x+1]$ has more than 3 elements - it has at least 3 elements, since $3(2 r-2 x+1)$ is a positive multiple of 3 ; if $3(2 r-2 x+1)=3$, then $2 x+2=6 r-4 x+2 \in \mathcal{H})$. Thus, in this case, $(2 x+2)+(6 r-2 x-4)=6 r-2=\ell_{g}$ would be a nongap, a contradiction. 
So, in any case, we arrive at a contradiction, and conclude that $6 r-5$ is a gap, which implies, by Lemma 3.4, that $3 \in \mathcal{H}$ and it is straightforward to see that $\mathcal{H}=3 \mathbb{N}+\mathcal{H}_{6 r-2}$.

Corollary 3.6. If $\mathcal{H}$ is a limit sparse semigroup with even Frobenius number $\ell_{g}=2 g-2 r$, then $\mathcal{H}$ is an Arf semigroup.

We now have a tight bound for the genus of a sparse semigroup with even Frobenius number, greatly improving that by Munuera, Torres and Villanueva $\left(g \leq 6 r-n_{1}\right.$ if $g \geq 4 r-1$ [MTV, Theorem 3.1]):

Corollary 3.7. Let $\mathcal{H}$ be a sparse semigroup of genus $g$ with even Frobenius number $\ell_{g}=2 g-2 r$. Then $g \leq 4 r-1$.

Proof. Suppose $\mathcal{H}$ is a sparse semigroup of genus $g=4 r+j, j \geq 0$, with $\ell_{g}=2 g-2 r$. Then, by Lemma 2.3, there exists a sparse semigroup $\widetilde{\mathcal{H}}$ of genus $\widetilde{g}=4 r-1$ and $\ell_{\widetilde{g}}=6 r-2$ such $\mathcal{H}$ is a subsemigroup of $\widetilde{\mathcal{H}}$. Theorem 3.5 tells us that $\widetilde{\mathcal{H}}=3 \mathbb{N}+\mathcal{H}_{6 r-2}$. By the construction of $\widetilde{\mathcal{H}}$ in the proof of Lemma 2.3, we see that $\{n \in \mathbb{N} ; n<6 r\} \cap \mathcal{H}=\{n \in \mathbb{N} ; n<6 r\} \cap \widetilde{\mathcal{H}}$; in particular, $3 \in \mathcal{H}$ and thus $6 r \in \mathcal{H}$, which contradicts the fact that $6 r=\ell_{4 r}=\ell_{g-j}$ is a gap of $\mathcal{H}$.

Let us now analyze some constraints on limit sparse semigroups having odd Frobenius number.

Theorem 3.8. Let $\mathcal{H}$ be a limit sparse semigroup with odd Frobenius number $\ell_{g}=2 g-(2 r+1)=$ $6 r+1$. If the multiplicity $n_{1}$ of $\mathcal{H}$ is even, then every nongap smaller then $\ell_{g}$ is even, and so $\mathcal{H}$ is r-hyperelliptic.

Proof. Assume there is an odd nongap $x \in \mathcal{H}, x<\ell_{g}$. Let $\tilde{n}$ be the largest odd nongap smaller than $\ell_{g}$. The interval $\left(\ell_{g}-n_{1}, \ell_{g}\right]$ contains a complete residue system of the integers module $n_{1}$, so $\tilde{n}$ satisfies $\ell_{g}-n_{1}<\tilde{n}<\ell_{g}$. By construction, $\tilde{n}+1$ and $\tilde{n}+2$ are consecutive gaps. So, since $\mathcal{H}$ is sparse, there are less than $n_{1} / 2$ nongaps in the interval $\left(\ell_{g}-n_{1}, \ell_{g}\right]$, thus there are at least $2 r-n_{1} / 2+1$ positive nongaps smaller than $\ell_{g}-n_{1}$. Define $\Gamma:=\left\{n \in \mathcal{H} ; 0<n<\ell_{g}-n_{1}\right\}$. Being $\tilde{n}+1$ and $\tilde{n}+2$ consecutive gaps, for each $n \in \Gamma, \tilde{n}+1-n$ and $\tilde{n}+2-n$ are also consecutive gaps. But in the interval $\left[1, n_{1}-1\right]$ there are at least $n_{1} / 2-1$ single leaps distinct from each $(\tilde{n}+1-n, \tilde{n}+2-n)$. By considering also the single leap $(\tilde{n}+1, \tilde{n}+2)$ we already count a number of $\left(2 r-n_{1} / 2+1\right)+\left(n_{1} / 2-1\right)+1=2 r+1$ single leaps, a contradiction. Now, the odd gaps are all the odd numbers between 1 and $\ell_{g}=6 r+1$, and so there are $3 r+1$ odd gaps and $r$ even gaps, then $\mathcal{H}$ is $r$-hyperelliptic.

Theorem 3.9. Let $\mathcal{H}$ be a limit sparse semigroup with odd Frobenius number $\ell_{g}=2 g-(2 r+1)=$ $6 r+1$. If the multiplicity $n_{1}$ of $\mathcal{H}$ is odd, then $\mathcal{H}$ is one of the following:

1. $\mathcal{H}=3 \mathbb{N}+\mathcal{H}_{6 r+1}$;

2. $\mathcal{H}=\langle 2 j+1 ; j \in \mathbb{N}, r \leq j \leq 2 r-1\rangle \cup \mathcal{H}_{6 r+1}$, with $r>1$.

Proof. Let us first show that, if $6 r-2 \notin \mathcal{H}$, then $3 \in \mathcal{H}$ (and so $\mathcal{H}=3 \mathbb{N}+\mathcal{H}_{6 r+1}$ ). If $6 r-2 \notin \mathcal{H}$, then, since $S=D$ and because of Lemma 3.2. there are $2 r-1$ nongaps in the interval [1,6r-2]. Then for each such nongap $n \in \mathcal{H}$, the consecutive numbers $6 r-2-n$ and $6 r-1-n$ are gaps, which produces $2 r-1$ single leaps. Notice that the single leaps $(6 r-2-n, 6 r-1-n)$ are all disjoint, for every $n \in \mathcal{H}$, because of the sparse property. 
Suppose, by contradiction, that $3 \notin \mathcal{H}$. Thus $(1,2)$ and $(2,3)$ are single leaps, and $(6 r-2-$ $n, 6 r-1-n)$ are $2 r-1$ disjoint single leaps, for every $n \in \mathcal{H} \cap[1,6 r-2]$, and so at least one of the two single leaps $(1,2)$ and $(2,3)$ are not of this form. Hence, since $(6 r-2,6 r-1)$ is also a single leap, the number of single leaps is bigger than $2 r$, which is a contradiction.

Suppose now that $6 r-2$ is a nongap. So, there is an integer $x \geq 2$ such that the last single leap is $(6 r-2 x, 6 r-2 x+1)$. So, in the interval $[6 r-2 x+1,6 r+1]$, all odd numbers are gaps and all even numbers are nongaps.

First notice that it cannot be that $n_{1}<2 x+1$. Otherwise, $6 r+1-n_{1}$ would be an even gap and $6 r+1-n_{1}>6 r-2 x$, a contradiction. Then there are only two possibilities for $n_{1}$ :

(A) $n_{1}>2 x+1$;

(B) $n_{1}=2 x+1$.

Since the nongaps in the interval $[6 r-2 x+1,6 r+1]$ are the even numbers, there are $2 r-x$ positive nongaps smaller than $6 r-2 x$. For each one of them, say $n \in \mathcal{H} \cap[1,6 r-2 x-1]$, $6 r-2 x-n$ and $6 r-2 x+1-n$ are consecutive gaps, producing $2 r-x$ single leaps.

Let us consider each case separately:

If we assume (A), then $1,2,3, \ldots, n_{1}-1$ are consecutive gaps. The single leaps $(6 r-2 x-$ $n, 6 r-2 x+1-n)$ are all disjoint for every $n \in \mathcal{H}, n<6 r-2 x$. Then, among the single leaps in the interval $\left[1, n_{1}-1\right]$, there are at least $x$ different from the ones in $\{(6 r-2 x-n, 6 r-2 x+$ $1-n) ; n \in \mathcal{H}, n<6 r-2 x\}$. Since $(6 r-2 x, 6 r-2 x+1)$ is also a single leap, we have, so far, $x+(2 r-x)+1=2 r+1$ single leaps, which is a contradiction, because $S=2 r$.

Let us assume (B). Since $S=2 r$, there are exactly $x-1$ single leaps in the interval $\left[1, n_{1}-1\right]$ that are not in $\{(6 r-2 x-n, 6 r-2 x+1-n) ; n \in \mathcal{H}, n<6 r-2 x\}$. Thus, there are $x$ single leaps in the interval $[1,2 x]$ of the type $(6 r-2 x-n, 6 r-2 x+1-n)$, where $n \in \mathcal{H} \cap[1,6 r-2 x-1]$, which are necessarily $(1,2),(3,4), \ldots,(2 x-1,2 x)$. Hence $6 r-4 x+1, \ldots, 6 r-2 x-3,6 r-2 x-1 \in \mathcal{H}$. In particular, since $x \geq 2,6 r-2 x-3 \in \mathcal{H}$, and, by the sparse property, $6 r-4 x$ is a gap.

Now, consider the interval $[2 x+1,6 r-4 x]$ which has $6(r-x)$ integers and contains $2 r-x-x=$ $2 r-2 x$ nongaps. For each nongap $n \in \mathcal{H} \cap[2 x+1,6 r-4 x]$, we consider the consecutive gaps $6 r-2 x-n$ and $6 r-2 x+1-n$, which both belong to [2x-1, $6 r-4 x+1]$ (and thus are $2 r-2 x$ single leaps contained in this interval). Then, since $\mathcal{H}$ is sparse, the nongaps in the interval $[2 x+1,6 r-4 x]$ are necessarily the integers congruous to $2 x+1(\bmod 3)$. Hence, provided $r-x \neq$ 0 (in which case the interval $[2 x+1,6 r-4 x]$ has $6(r-x) \geq 6$ integer elements), we have $2 x+4 \in \mathcal{H}$. Thus, in this case, $(2 x+4)+(6 r-2 x-3)=6 r+1=\ell_{g}$ would be a nongap, a contradiction. Thus we must have $r-x=0$, i.e., $x=r$, and so $6 r-4 x+1=2 x+1$ and $6 r-2 x+2=4 x+2$. In this case, $\mathcal{H}=\{0\} \cup\{2 j+1 ; j \in \mathbb{N}, r \leq j \leq 2 r-1\} \cup\{2 j ; j \in \mathbb{N}, 2 r+1 \leq j \leq 3 r\} \cup\{n \in \mathbb{N} ; n \geq 6 r+2\}$, which is clearly the semigroup $\mathcal{H}=\langle 2 j+1 ; j \in \mathbb{N}, r \leq j \leq 2 r-1\rangle \cup \mathcal{H}_{6 r+1}$.

Remark 3.10. The semigroups of the form $\mathcal{H}=3 \mathbb{N}+\mathcal{H}_{6 r+1}$ are clearly Arf, but the semigroups of the form $\mathcal{H}=\langle 2 j+1 ; j \in \mathbb{N}, r \leq j \leq 2 r-1\rangle+\mathcal{H}_{6 r+1}$ with $r>1$ are not Arf, since $4 r-3,4 r-1 \in \mathcal{H}$, but 2. $(4 r-1)-(4 r-3)=4 r+1 \notin \mathcal{H}$.

Corollary 3.11. Let $\mathcal{H}$ be a sparse semigroup of genus $g$ with $\ell_{g}=2 g-(2 r+1)$. Then either $g \leq 4 r+1$, or all nongaps smaller than $\ell_{g}$ are even.

Proof. Suppose $\mathcal{H}$ is a sparse semigroup of genus $g=4 r+j=2(2 r+1)+(j-2), j \geq 2$, with $\ell_{g}=2 g-(2 r+1)$. Then, by Lemma 2.3 , there exists a sparse semigroup $\widetilde{\mathcal{H}}$ of genus $\widetilde{g}=4 r+1$ and $\ell_{\widetilde{g}}=6 r+1 \operatorname{such} \mathcal{H}$ is a subsemigroup of $\widetilde{\mathcal{H}}$. The previous theorems tell us that we have the following possibilities: 
(A) All nongaps of $\widetilde{\mathcal{H}}$ (and so of $\mathcal{H}$ ) smaller than $\ell_{\widetilde{g}}=6 r+1$ are even

In this case, by the construction of $\widetilde{\mathcal{H}}$ in the proof of Lemma 2.3, the gaps of $\mathcal{H}$ larger than $6 r$ are given by a sequence of double leaps starting at $6 r+1$, and so all the remaining nongaps of $\mathcal{H}$ smaller than $\ell_{g}$ are even.

(B) $\tilde{\mathcal{H}}=3 \mathbb{N}+\mathcal{H}_{6 r+1}$

Here, by the construction of $\widetilde{\mathcal{H}}$ in the proof of Lemma 2.3. we see that $\{n \in \mathbb{N} ; n<$ $6 r+2\} \cap \mathcal{H}=\{n \in \mathbb{N} ; n<6 r+2\} \cap \widetilde{\mathcal{H}}$; in particular, we have $6 r+3 \in \mathcal{H}$, which contradicts the fact that $6 r+3=\ell_{4 r+2}=\ell_{g-j+2}$ is a gap of $\mathcal{H}$.

(C) $\widetilde{\mathcal{H}}=\langle 2 j+1 ; r \leq j \leq 2 r-1\rangle \cup \mathcal{H}_{6 r+1}$.

Since $2 r+1 \in \mathcal{H}$ we have $6 r+3=3(2 r+1) \in \mathcal{H}$ - which also contradicts the fact that $6 r+3=\ell_{4 r+2}=\ell_{g-j+2}$ is a gap of $\mathcal{H}$.

\section{On sparse Weierstrass semigroups}

One of the main applications of numerical semigroups is the study of Weierstrass points on curves. Having dealt (and classified limit) sparse semigroups, it is natural to ask whether those are realized as a Weierstrass semigroup.

By a curve, we mean a smooth projective curve defined over an algebraically closed field of characteristic zero. If $\mathcal{X}$ is a curve and $P \in \mathcal{X}$ is a point of $\mathcal{X}$, then the Weierstrass semigroup $H(P)$ of the pair $(X, P)$ consists of the integers $n$ for which there does exists a meromorphic function on $\mathcal{X}$ with pole divisor $n P$, i.e.

$$
H(P):=\left\{n \in \mathbb{N} ; \operatorname{dim} H^{0}\left(\mathcal{X}, O_{\mathcal{X}}((n-1) P)\right)<\operatorname{dim} H^{0}\left(\mathcal{X}, O_{\mathcal{X}}(n P)\right)\right\}
$$

It is clear that $H(P)$ is a numerical semigroup. Now, it follows from Riemann-Roch Theorem that the gap sequence of $H(P)$ is the sequence of orders of vanishing of the holomorphic differentials of $\mathcal{X}$ at $P$. In particular, the genus of $H(P)$ is equal to the genus of the curve $\mathcal{X}$. A numerical semigroup is a Weierstrass semigroup if it is realized as Weierstrass semigroup of some pair $(X, P)$. A point on a curve is a Weierstrass point if its associated Weierstrass semigroup is different from $\mathcal{H}_{g}$, where $g$ is the genus of the curve.

It is known, from Rim-Vitulli [RV], that the negatively graded semigroups are only the ordinary, hyperordinary and those semigroups of multiplicity $m>1$ having precisely one gap between $m$ and $2 m$. By a theorem of Pinkham $[\mathrm{P}]$, we know that a monomial curve associated to a negatively graded semigroup can be smoothed. In particular, a (hyper)ordinary semigroup is a Weierstrass semigroup. Thus, from those works and Theorem 3.5, we get:

Corollary 4.1. Let $\mathcal{H}$ be a limit sparse semigroup of genus $g$. If the Frobenius number $\ell_{g}$ is even, then $\mathcal{H}$ is a Weierstrass semigroup;

It is clear that if $\mathcal{H}$ is a limit sparse semigroup with odd Frobenius number of the type $\mathcal{H}=3 \mathbb{N}+\mathcal{H}_{6 r+1}$ (see Theorem 3.9), then $\mathcal{H}$ is also a Weierstrass semigroup.

Corollary 4.2. Let $\mathcal{H}^{\prime}$ be any numerical semigroup of genus $r$. Consider $\mathcal{H}:=2 \mathcal{H}^{\prime} \cup \mathcal{H}_{6 r+1}$. Then $\mathcal{H}$ is a limit sparse semigroup with odd Frobenius number with even multiplicity (see Theorem 3.8). Reciprocally, every semigroup satisfying the hypothesis of Theorem 3.8 arises in this way. Moreover, $\mathcal{H}$ is Arf if and only if $\mathcal{H}^{\prime}$ is Arf. 
Proof. It is clear that if $\mathcal{H}^{\prime}$ is a semigroup of genus $r$, then the Frobenius number of $\mathcal{H}:=$ $2 \mathcal{H}^{\prime} \cup \mathcal{H}_{6 r+1}$ is $6 r+1$. The gaps of $\mathcal{H}$ are all the odd integers in [1, $\left.6 r+1\right]$ and the even numbers $2 m$ where $m$ is a gap of $\mathcal{H}^{\prime}$. Thus the genus of $\mathcal{H}$ is $4 r+1$. The sparse condition follows from the construction of $\mathcal{H}$. On the other hand, let $\mathcal{H}$ be a sparse semigroup of genus $g=4 r+1$, with Frobenius number $\ell_{g}=2 g-(2 r+1)$, and even multiplicity $n_{1}$. Thus Theorem 3.8 ensures that all nongaps smaller than $\ell_{g}=6 r-1$ are even. The odd gaps are all the odd numbers between 1 and $\ell_{g}=6 r+1$. So there are $3 r+1$ odd gaps and $r$ even gaps. Thus $\mathcal{H} \cap\left[1, \ldots, \ell_{g}\right]=$ $\left\{2 n_{1}, 2 n_{2}, \ldots, 2 n_{2 r}\right\}$. We consider the set $\mathcal{H}^{\prime}:=\left\{0, n_{1}, \ldots, n_{2 r}, n_{2 r}+1, n_{2 r}+2, \ldots\right\}$. Since $\mathcal{H}$ is additively closed, $\mathcal{H}^{\prime}$ a semigroup. An integer is a gap of $\mathcal{H}^{\prime}$ if and only if $2 n$ is a gap of $\mathcal{H}$. Thus the genus of $\mathcal{H}^{\prime}$ is $r$. The Arf condition follows from the construction of $\mathcal{H}^{\prime}$. We notice that the last gap in $\mathcal{H}^{\prime}$ is at most $2 r-1$, and so the last even gap of $\mathcal{H}$ is at most $4 r-2$.

Following the same steps of the above Corollary, we get:

Corollary 4.3. Let $\mathcal{H}$ be a semigroup as in Corollary 3.11 with $g>4 r+1$. Then $\mathcal{H}$ is obtained in the following way: take any numerical semigroup $\mathcal{H}^{\prime} \subset \mathbb{N}$ of genus $r$, and set $\mathcal{H}:=2 \mathcal{H}^{\prime} \cup$ $\mathcal{H}_{2 g-2 r-1}$.

Proof. If $\mathcal{H}$ is a numerical semigroup as in Corollary 3.11, with $g>4 r+1$, then its odd gaps are all the odd numbers between 1 and $\ell_{g}=2 g-(2 r+1)$. So, there are $g-r$ odd gaps and $r$ even gaps. Thus $\mathcal{H}:=2 \mathcal{H}^{\prime} \cup \mathcal{H}_{2 g-2 r-1}$ where $\mathcal{H}^{\prime}$ is a numerical semigroup of genus $r$. Note that the last gap of $\mathcal{H}^{\prime}$ is at most $2 r-1$, and so the last even gap of $\mathcal{H}$ is at most $4 r-2$.

As can be noted of from the last two corollaries above, and the Corollary 3.11, we may expect that some sparse semigroups with odd Frobenius number arise as a double covering of a genus $r$ curve. We recall that Torres [T] characterized $r$-hyperelliptic curves of genus $g$ which arise as a double covering of a genus $r$-curves under the assumption $g \geq 6 r+4$. Gathering the Corollary 4.3 and the comment after the proof of Theorem A of [T] we get:

Corollary 4.4. Let $\mathcal{H}$ a numerical sparse semigroup of genus $g \geq 6 r+4$ and Frobenius number $\ell_{g}=2 g-(2 r+1)$. If $\mathcal{H}$ is a Weierstrass semigroup, then it arises as a double covering of a genus r-curve. In this case, we have $\mathcal{H}:=2 \mathcal{H}^{\prime} \cup \mathcal{H}_{2 g-2 r-1}$, where $\mathcal{H}^{\prime}=\{n / 2 \mid n \in \mathcal{H}$ is even $\}$ is a Weierstrass semigroup of genus $r$.

Question 4.5. Let $\mathcal{H}^{\prime} \subset \mathbb{N}$ any Weierstrass semigroup of genus $r$, and $g \geq 4 r+1$. Is the semigroup $\mathcal{H}:=2 \mathcal{H}^{\prime} \cup \mathcal{H}_{2 g-2 r-1}$ always Weierstrass?

Question 4.6. Let $\mathcal{H}$ be a sparse Weierstrass semigroup with odd Frobenius number $\ell_{g}=2 g-$ $(2 r+1)$ and $g \geq 4 r+1$ such that the multiplicity $n_{1}$ of $\mathcal{H}$ is even. Is the semigroup $\mathcal{H}^{\prime}=\{n / 2 \mid$ $n \in \mathcal{H}$ is even $\}$ always Weierstrass?

Remark 4.7. If the answer to Problem A of [Kom] is affirmative then the answer to this last question is also affirmative (indeed it would be enough that there were no numerical semigroup belonging to the box numbered by viii) in [Kom]).

Question 4.8. Is the limit sparse semigroup $\mathcal{H}=\langle 2 j+1 ; j \in \mathbb{N}, r \leq j \leq 2 r-1\rangle \cup \mathcal{H}_{6 r+1}$ Weierstrass for every $r>1$ ?

\section{Acknowledgements}

The authors warmly thank Prof. Luciane Quoos (Universidade Federal do Rio de Janeiro) for introducing sparse semigroups to them. 


\section{References}

\section{References}

[Arf] C. Arf, Une interpretation algébrique de la suite des ordres de multiplicité d'une branche algébrique, Proc. London Math. Soc., 50 (1949), 256 - 287

[BC] E. Ballico, A. Del Centina, Ramification Points of double covering of curves and Weierstrass Points, Annali di Matematica Pura ed Applica, 178, (1999), 293-313.

[BDF] V. Barucci, D. E. Dobbs, M. Fontana, Maximality properties in numerical semigroups and applications to onedimensional analytically irreducible local domains, Mem. Amer. Math. Soc. 125, 1997.

[Lip] J. Lipman, Stable ideal and Arf semigroups, Amer. J. Math. 97 (1975), 791 - 813.

[Kom] J. Komeda, A generalization of Weierstrass semigroups on a double covering of a curve, Languages, Computations, and Algorithms in Algebraic Systems - Kyoto University Research Information Repository 1655 (2009), 124 - 131. Available at http://www.kurims.kyoto-u.ac.jp/ kyodo/kokyuroku/contents/pdf/1655-15.pdf

[MTV] C. Munuera, F. Torres, J. Villanueva, Sparse Numerical Semigroups, Lecture Notes in Computer Science: Applied Algebra, Algebraic Algorithms and Error-Correcting Codes, 5527, 23 - 31, Springer-Verlag Berlin Heilderberg (2009).

[O] G. Oliveira, Weierstrass semigroups and the canonical ideal of non-trigonal curves, Manuscripta Math., 71 (1991), $431-450$.

[P] H. Pinkham, Deformations of algebraic varieties with $G_{m}$-action, Astérisque 20 (1974), 1-131.

[RV] D.S. Rim and M.A. Vitulli, Weierstrass points and monomial curves, J. Algebra 48 (1977) 454-476.

[RGB] J.C. Rosales, P. A. García-Sánchez, J. I. García-García, M. B. Branco, Arf Numerical Semigroups, Journal of Algebra 276 (2004), 3 - 12.

[T] F. Torres, Weierstrass points and double coverings of curves with applications: Symmetric numerical semigroups which cannot be realized as Weierstrass semigroups, Manuscripta Math 83 (1994), 39 - 58.

[T2] F. Torres, On $\gamma$-Hyperelliptic Numerical Semigroups, Semigroup Forum 55 (1997), 364 - 379. 